\title{
DIE ERSTELLUNG DER LERNMEDIEN MIT DER HILFE MACROMEDIA AUTHORWARE 7.0 FÜR DAS LERNEN DER NEUEN DEUTSCHEN RECHTSCHREIBUNG
}

\author{
Fitri Lasmaria Br Sihite \\ Risnovita Sari \\ Herlina JP Harahap
}

\begin{abstract}
AUSZUG
Das Ziel dieser Untersuchung ist es, um die Lernmedien mit der Hilfe Macromedia Authorware 7.Ofür das Lernen der neuen deutschen Rechtschreibung aus dem Buch „Die neue Rechtschreibung *kurz und einfach” von Christian Stang zu erstellen. In dieser Untersuchung wird die deskriptive qualitative Methode angewendet. Der Prozess der Erstellung der Lernmedien mit der HilfeMacromedia Authorware 7.0 Software für das Lernen der neuen deutschen Rechtschreibung aus dem Buch „Die neue Rechtschreibung *kurz und einfach" von Christian Stang besteht aus der Erklärung der Phasen von Richey und Klein. Darunter sind: (1) Die Planung, (2) Die Herstellung des Produkts und (3) Die Bewertung und Verbesserung. Die Daten in dieser Untersuchung sind Bilder und Texte mit dem Thema „Die neue Rechtschreibung *kurz und einfach". Diese Untersuchung wird in der Sprachleseraum/Bibliothek an der Fakultät für Sprachen und Kunst an der Staatlichen Universität Medan durchgeführt. Das Ergebnis der Erstellung der Lernmedien mit der HilfeMacromedia Authorware 7.0für das Lernen der neuen deutschen Rechtschreibung aus dem Buch „Die neue Rechtschreibung *kurz und einfach” von Christian Stang (Die Laut-Buchstaben-Zuordung, Die Fremdwortschreibung, Die Worttrennung am Zeilenende, Die Getrennt- und Zusammenschreibung, Die Schreibung mit Bindestrich und Die Groß- und Kleinschreibung), Haupseite, Benutzeridentitätseite, Hauptmenü (Einleitung, Materie, Übungen, Quelle, Biographie und Ausgang). Hoffentlich kann diese Abschlussarbeit nützlich sein, um unser Wissen zur Wissenschaft über Lernsoftware, vor allem Deutschlernenden anzureichern.
\end{abstract}

Schlüsselwörter: Erstellung von Lernmedien, Macromedia Authorware 7.0, Die neue Rechtschreibung

\section{EINLEITUNG}

Die Lernmedien sind in der Universität ein integraler Bestandteil des Lernens, denn nur durch Üben lernt man am besten. Dazu sind Lernmedien als Lernressourcen erforderlich. Die Nutzung der Medien beim Lernen verbessert die Ergebnisse des Lernens und steigert die Lernmotivation der Studenten. Die Angemessenheit der Verwendung von Lehrmedien ist untrennbar mit dem Verständnis von Vielfalt und den Eigenschaften der Medien verbunden. Die Lernmedien können verwendet werden, um die Interaktion zwischen dem Dozenten und den Studenten zu erhöhen. Aber es gibt viele Hindernisse die im Unterricht auftreten können, zum Beispiel:Die 
Varianten des Materials zum Lernen der neuen deutschen Rechtschreibung sind zu begrenzt, sodass die Studenten nur eine geringe Lust zum Deutschlernen haben.

Im Allgemeinen benutzen Dozenten Lehrbücher und Wörterbücher im Unterricht. Basierend auf dieser Beobachtung ist die Verwendung des Lernmediums noch nicht effektiv, vor allem beim Thema „Die neue Rechtschreibung”, denn es wird nur das Lehrbuch benutzt. Es wäre besser, wenn verschiedene Medien benutzt werden würden, denn der Unterricht wäre interessanter, und die Studenten bekämen viel mehr Informationen.

Daneben kann die Unfähigkeit der Dozenten bei derTechnologieverwendung beim Lernprozessauchein Hindernis sein. Daher müssenneue Lernmedien erstellt werden. Wissenschaft und Technologie kann beimLernprozess sehr nützlich sein. Eine Technologie, mitderen hilfe Lernmedien erstellt werden können, ist die SoftwareMacromedia Authorware 7.0.

Macromedia Authorware 7.0 ist eine visuelle Programmiersoftware, die mit Icon undflowline Methoden arbeitet. Mit Macromedia Authorware 7.0 können Lernmedien in Form von Texten, Bildern,Tönen, undAnimationen direkt angezeigt werden. Solche modernen Lernmedien sind nach wie vor aufgrund der geringen Mediennutzung im Unterricht, sodass sie schwer $\mathrm{zu}$ bekommen sind. Es gibt alsoeinen Fehlerin der Medienentwicklung. Daher wird die Erstellung dieses Lernmediums, für das Lernender ,,neuen deutschen Rechtschreibung”für die meisten Studentensehr hilfreich sein, um die Sprachregeln und ihre richtige Anwendung zu lernen.

Im Jahr 2007 (Mittwoch, den 1. August) wurde die neue Rechtschreibung in Deutschland eingeführt. (Mit dem Link mobil.derstandard.at/2978298/Ab1-Augustneue-Rechtschreibung-in-Deutschland---2008-in-Oesterreich, gelesen am 27. Februar 2017 um 10.00). Die wichtigsten Neuerungen der deutschen Rechtschreibung. Die zehn grundsätzlichen Änderungen betreffen: (1) die Anwendung von ss und $\beta$; (2) die Großschreibung und (3) die Kleinschreibung; (4) die einheitliche Schreibung gleicher Wortstämme; (5) die Rechtschreibung von Fremdwörtern; (6) die Getrenntschreibung und (7) die Zusammenschreibung; (8) die Worttrennungen am Zeilenende sowie (9) die Anrede in Briefen und (10) die Kommasetzung(vgl. Dittmann2008: 13). 
Am 18. Juli 2017 wurde eine Umfragemit 20 Studenten in der Deutschabteilung gemacht. Aus der Beobachtung und eine kleiner Untersuchung die am 18. Juli 2017 mit 20 Studenten in der Deutschabteilung an der staatlichen Universität Medan (UNIMED) durchgeführt wurde, folgt: Es gibt Fehler bei der Verwendung von $\beta$ (Eszett) und ss (Doppel-s), der Getrenntschreibung und Zusammenschreibung, der Worttrennung am Zeilenende, der Großschreibung und Kleinschreibung, der Verdoppelung von Konsonanten, dem Zusammentreffen dreier gleicher Buchstaben, und der Fremdwortschreibung.

Aus dem Ergebnis der Umfrage ergibt sich, dass es noch einige Fehler beim Lernen der neuen deutschen Rechtschreibung gibt. Von der obigen Umfrage kann gesehen werden, dass die Studenten noch Schwierigkeiten haben, die neue Rechtschreibung anzuwenden. Die Studenten müssen wissen, dass sich die Schreibweise einer Sprache verändern kann. Es ist auf die Einführung der neuen Rechtschreibregeln im Deutsch zu achten.

Macromedia Authorware 7.0 kann im Lernprozess verwendet werden, weil es ein effektives, effizientes, und zugängliches Lernmedium für die Studenten ist. Die Software Macromedia Authorware 7.0 hat viele Vorteile, unter anderem kann man eine multimediale-Präsentationen in einigen Formen direkt machen, zum Beispiel die Animation, die Töne, und die Bilder. Die Lernmedien in dieser Untersuchung enthalten Erklärungen zur neuen deutschen Rechtschreibung und dazu passende Übungen. Mit der Software Macromedia Authorware 7.0 wird der Lernprozess interessanter, effektiver und praktischer, weil die Verwendung dieser Software einfach ist, sodass die Studenten die neue deutsche Rechtschreibung leichter verstehen können.

Im Folgenden wird eine Untersuchung über die Erstellung der Lernmedium mit der Hilfe von Macromedia Authorware 7.0 für das Lernen der neuen deutschen Rechtschreibung durchgeführt.

\section{THEORETISCHE GRUNDLAGE}

\section{Der Begriff der Erstellung}

Borg und Gall(in Sugiyono 2016:28)stellen fest, dass Untersuchung und Erstellung einProzess oder eine Methode ist, um Produkte zu entwickeln.Richey und 
Klein (in Sugiyono 2016:30) stellen fest, dass der Schwerpunkt auf dem Design und der Erstellung von Produkten liegt. Dieser Prozess kann von Anfang bis Ende analysiert werden.Richey und Klein (in Sugiyono 2016:39) behaupten ,The focus of Design and Development Research can be on front-end analysis, Planning, Production, and Evaluation (PPE)“.Das Richey und Klein-Modell ist einfach und systematisch in der Verwendung.

\section{Der Begriff des Lernmediums}

Daryanto (2010:4) stellt fest, dass das Medium ist ein Mittel oder Instrument ist, dass im Lernprozess helfen kann. Im Lernprozess werden Medien benutzt, um visuelle und verbale Informationenzu verarbeiten. Heinich (in Daryanto 2010:4) behauptet ebenfalls, dass ein Medium ein Vermittler vom Absender zumEmpfänger ist. Criticos (in Daryanto 2010:4) stellt fest, dass das Medium eine Komponente der Kommunikation ist. Im Lernprozess ist das Medium ein integraler Bestandteildes Lernsystems.

\section{Die Software Macromedia Authorware 7.0}

Die SoftwareMacromedia Authorware 7.0 ist eine visuelle Programmiersoftware, die mitIcon und Flowline Methoden arbeitet. Macromedia Authorware hat ein einzigartiges und interessantes Grundkonzept. Die Erstellung von Anwendungen mit Authorware funktioniert so wie die Erstellung eines Diagramms in Microsoft Word. Die Verwendung von Symbolen mit flowline Authorware ist nicht nur einfach ein 'Bild'-Symbol, sondern hat auch eine einzigartige Form und Funktion im Einklang mit der Ordnung des Szenarios (vgl. Tim Wahana Komputer 2004:37).Setyono et,al.(2008:1) stellt fest, dass Macromedia Authorwareein Programm ist, das eine umfassende Einrichtung ist.

\section{Die neue deutsche Rechtschreibung}

Dittmann (2008:7) sagt: „Die neue Rechtschreibung ist neu in der Geschichte der deutschen Orthografie, eine Reihe von Regeln, die es ermöglichen, auch ohne den Blick ins Wörterbuch korrekt zu schreiben.” Sekawan (vgl. 2007:1) erklärt, dass die Rechtschreibung ein ganzes System von Lauten und Regeln ist, dass dabei hilft, eine gewisse Einheitlichkeit zu erreichen.Holstfügt hinzu: „Die Einheitlichkeit ist für eine Rechtschreibung ein hohes Gut. Sie kennzeichnet jede modern entwickelte 
Schriftsprache. In diesem Zusammenhang ist es wichtig zu erkennen, dass sich keine neue Einheitlichkeit mit der neuen Rechtschreibung einstellen wird, denn die alte wird ja weiterhin benutzt, und das gerade von der intellektuellen Elite, darunter Schriftstellern,Sprachwissenschaftlern, und besseren Zei tungen“.

\section{UNTERSUCHUNGSMETODE}

Diese Untersuchung ist eine Erstellungsuntersuchung (Research \& Development). Es wird ein Lernmedium mithilfe der SoftwareMacromedia Authorware 7.0 erstellt. Es wird dafür die deskriptive qualitative Methode benutzt.

\section{Die Quelle der Daten}

Die Datenquellevon dieser Untersuchung ist das Deutschbuch „Die neue Rechtschreibung *kurz und einfach“" von Christian Stang aus dem Jahr 2014.

\section{Die Skizze der Untersuchung}

In dieser Untersuchung wird die Theorie von Richey und Klein verwendet, das aus drei Schritten besteht. Diese sind: 1) die Planung, (2) die Herstellung des Produkts, und (3) die Bewertung und Verbesserung.

\section{ERGEBNIS DER UNTERSUCHUNG}

\section{Der Prozess der Erstellung}

\section{Die Planung}

Die Planung ist die Phase der Bedarfsanalyse. In diesem ersten Schritt wurde eine Umfrage und eine Beobachtung durchgeführt. Am 18. Juli 2017 wurde eine Umfrage mit 20 Studenten in der Deutschabteilung gemacht. Aus der Beobachtung und eine kleiner Untersuchung am 18. Juli 2017, die 20 Studenten in der Deutschabteilung an der staatlichen UniversitätMedan (UNIMED) durchgeführt haben, folgt:Fehler bei der Verwendung von $\beta$ (Eszett) und ss (Doppel-s), der Getrenntschreibung und Zusammenschreibung, der Worttrennung am Zeilenende, der Großschreibung und Kleinschreibung, der Verdoppelung von Konsonanten, dem Zusammentreffen dreier gleicher Buchstaben, und derFremdwortschreibung.Aus dem Ergebnis der Umfrage ergibt sich, dass es noch einige Fehler beim Lernen der neuen deutschen Rechtschreibung gibt.

Das Thema der Rechtschreibung aus dem Buch von Christian Stang in dieser Untersuchung wird nur bis zum sechsten Thema diskutiert, weil das nächste Thema ein neues Thema ist, nämlich: Zeichensetzung. 
In dieser Phase macht die Verfasserin eine Planung. Nachdem alle Materialien gesammelt wurden, wird das Material geordnet. Das Thema „Die neue Rechtschreibung *kurz und einfach" handelt von: 1. Die Laut-BuchstabenZuordnung, 2. Die Fremdwortschreibung, 3.Die Worttrennung am Zeilenende, 4. die Getrennt- und Zusammenschreibung, 5. die Schreibung mit Bindestrich, 6. die Großund Kleinschreibung.

\section{Das Design}

Das Design des Lernmediums wird mit der Macromedia Authorware 7.0 entworfen, um ein interessantes Lernmedium zu erstellen. Das Design wird an das Thema „Die neue Rechtschreibung kurz und einfach” angepasst, das in der Macromedia Authorware 7.0integriert wird. Alle Teile in der Macromedia Authorware 7.0können entworfen werden, um ein interessantes Lernmedium zu erstellen. Das Lernmedium wird interessant, wenn Bilder und Texte integriert werden.

\section{Die Herstellung}

Der zweite Schritt in dieser Untersuchung ist die Herstellung des Produkts. Der Erstellungsprozess der Lernmedien mit der Hilfe Macromedia Authorware 7.0 gliedert sich in drei Phasen, nämlich die Vorproduktion, Produktion und Postproduktion.

\subsection{Vorproduktion}

Diese Phase beginnt mit der Vorbereitung der Materialien zur Herstellung der Lernmedien. Diese sind die benötigen Materialien bei der Herstellung, zum Beispiel der Computer/Laptop,SoftwareMacromedia Authorware 7.0, unterstützende Materialien wie Bilder und Texte,die beim Lernprozess unterstützen können. Die Vorbereitung beginnt mit der installierung des Macromedia Authorware 7.0 auf dem Computer/Laptop.

\subsection{Produktion}

In dieser Phase wird die Erstellung der Lernmedien mit der Hilfe Macromedia Authorware 7.0 gemacht.

\subsection{Post-produktion}

Dies ist der Schritt, in dem die erstellten Lernmedien mit der Hilfe Macromedia Authorware 7.0, im die CD's gebrannt werden. 


\section{Die Bewertung und Verbesserung}

Das ist die letzte Phase, um ein interessante Lernmedium zu erstellen. In dieser Phase wurde schon das Lernmedium mit der Hilfe von Macromedia Authorware 7.0 Software erstellt. Dann werden die Prüfungund die Validierung durchgeführt. So kann herausgefunden werden,ob das Lernmedium mitMacromedia Authorware 7.0 Software schon gut genugist oder noch verbessert werden sollte. Dieses Lernmedium wird von einem Experten geprüft. Die Expertensind eine Dozentin, ein deutscher Muttersprachler und einem Designexperten. Die Experten haben einen pädagogischen Hintergrund.

\section{Das Ergebnis der Erstellung}

Das Design des Lernmediums ist vielfältig und kann auf unterschiedliche Weise entworfen werden. Die Hauptseite (Cover) des Lernmediums ist „Die neue Rechtschreibung kurz und einfach". Auf der Hauptseite gibt es eine Titelseite und Information über die Verfasserin. Die Hauptseite sieht aus wie ein Einband, der mit einem interessanten Layout erstellt wird. Die Farbe der Hauptseite ist wei $\beta$, rot, grün, gelb, orange und schwarz. Für die Art der Buchstabe wird "Times New Roman" und “Maiandra GD”.Auf der zweiten Seiten gibt es die Benutzeridentitätseite (Anmelden). Auf der dritten Seiten gibt es das Hauptmenü. Das Hauptmenü ist ein Menü, das von den Studenten bedient werden kann. Es kann Einleitung, Materie, Übungen, Quelle, Biographie und Ausgang gewählt werden. In der Einleitung gibt es eine kurze Erklärung für das Lernen der neuen Rechtschreibung. Die Materie enthält die Darstellung und die Information über die Materie. Das heisst das Thema „Die neue Rechtschreibung kurz und einfach", was die Themen umfasst: 1. Die LautBuchstaben-Zuordnung, 2. Die Fremdwortschreibung, 3.Die Worttrennung am Zeilenende, 4. die Getrennt- und Zusammenschreibung, 5. die Schreibung mit Bindestrich, 6. die Gro $\beta$ - und Kleinschreibung. In Übungen gibt es Aufgabe für die Studenten. In der Quelle können die Studenten die Quelle (die Information des Buch) dieser Untersuchung lesen. In Biographie finden sich Informationen bezüglich der Lernmedienerstellerin. In Ausgang kann man sich Abmelden. Dieses Lernmediums verwendet Bilder und Texte. Die Präsentation bei der Macromedia Authorware 7.0wird mit den umfassenden Erklärungenmit den Texten und Bildern mitdem Thema „Die neue Rechtschreibungkurz und einfach” dargestellt. Die Macromedia Authorware 7.0 kann Bilder und Texte erfassen. Mit diesen Elementen können interessante Lernmedien erstellt werden. 


\section{Die Diskussion}

Basierend auf den Ergebnissender Erstellung der Lernmedien mit der HilfeMacromedia Authorware 7.0 Software für das Lernen der neuen deutschen Rechtschreibung wurde zusammengefasst, dass die Daten in dieser Untersuchung aus dem Buch „Die neue Rechtschreibung *kurz und einfach” von Christian Stang kommen.In dieser Untersuchung wird die Theorie von Richey und Klein benutzt. Diese Theorie besteht aus drei Phasen, nämlich: (1) die Planung, (2) die Herstellung des Produkts und (3) die Bewertung und Verbesserung. In diesem Kapitel wird jede Phase mit ihrem Ergebnis erklärt. Bei der Planung wurde eine Umfrage und eine Beobachtung durchgeführt. Aus der Beobachtung und eine kleiner Untersuchung am 18. Juli 2017 mit 20 Studenten in der Deutschabteilung an der staatlichen Universität Medan (UNIMED) durchgeführt, folgt: Fehler bei der Verwendung von $\beta$ (Eszett) und ss (Doppel-s), der Getrenntschreibung und Zusammenschreibung, der Worttrennung am Zeilenende, der Großschreibung und Kleinschreibung, der Verdoppelung von Konsonanten, dem Zusammentreffen dreier gleicher Buchstaben, und der Fremdwortschreibung. Aus dem Ergebnis der Umfrage ergibt sich, dass es noch einige Fehler beim Lernen der neuen deutschen Rechtschreibung gibt.

Die Präsentation bei der Macromedia Authorware 7.0wird mit umfassenden Erklärungen mit Texten und Bildern mitdem Thema „Die neue Rechtschreibung kurz und einfach" dargestellt. Das Design wird mit Bildern und Texten mit dem Material „Die neue Rechtschreibung *kurz und einfach” realisiert. Das Thema „Die neue Rechtschreibung kurz und einfach" wurde schon in der Macromedia Authorware 7.0 Software realisiert. Danach wird in der Erstellung schon das Lernmedium mit der Hilfe Macromedia Authorware 7.0 Software erstellt.

Der Erstellungsprozess der Lernmedien mit der Hilfe Macromedia Authorware 7.0 gliedert sich in drei Phasen, nämlich die Vorproduktion, Produktion und Postproduktion. Die Vorproduktion beginnt mit der Vorbereitung der Materialien zur Herstellung der Lernmedien. Dies sind die benötigen Materialien bei der Herstellung, Computer/Laptop,die SoftwareMacromedia Authorware 7.0, unterstützende Materialien wie Bilder und Texte, die beim Lernprozess unterstützen können. Die Produktionder Erstellung der Lernmedien erstellt basierend auf Icon und Flowline erzielt worden. Die Herstellung von Lernmedien erfolgt mit Macromedia Authorware 7.0. Die Postproduktion, bei der erstellten Lernmedien mit der Hilfe Macromedia Authorware 7.0, auf die CD's gebrannt wird. Es wird eine Validierung durchgeführt. 
So kann herausgefunden werden, ob die Lernmedien mitMacromedia Authorware 7.0 schon gut genug sind oder noch verbessert werden sollten. Der Experte hat das LernmediumHilfe Macromedia Authorware 7.0 Software schon korrigiert. Dann hat der Experte das Lernmedium benotet: von 8 Kategorien der Aspekt der Evaluation gibt es die Note 4für sechsAspekte und der Expert gibt die Note 3 für zwei Aspekte, sodass die Note der Lernmedien mit der Hilfe Macromedia Authorware 7.0 ist 93,75 (sehr gut).

In dieser Untersuchung gibt es die Vorteile und Nachteile, nämlich: Vorteile von dieser Untersuchung sind (1) die Studenten können die neue Rechtschreibung leichter verstehen, (2) daher wird die Untersuchung, insbesondere für das Lernen der neuen Rechtschreibung für die meistern Studenten sehr hilfreich sein, um die Sprachregeln und richtige Anwendung zu lernen, (3) mit der Untersuchung lernen Studenten die schriftliche Information für das Lernen der neuen deutschen Rechtschreibung, besser zu verstehen. Die Nachteile von dieser Untersuchung: In dieser Untersuchung wird des Lernmediums nur Bilder und Texte gemacht. Basierend auf der obigen Erklärung kann zusammengefasst werden, dass die Erstellung der Lernmedien mit der Hilfe Macromedia Authorware 7.0 für das Lernen der neuen deutschen Rechtschreibungaus dem Buch,,Die neue Rechtschreibung *kurz und einfach" von Christian Stang mit der Richey und Klein Theorie sehr gut ist, sodass die Dozenten oder die Studenten das Lernmedium benutzen können.

\section{SCHLUSSFOLGERUNG}

Die Schlussfolgerungen dieser Untersuchung sind:

1. Der Prozess der Erstellung der Lernmedienmit der Hilfe von Macromedia Authorware 7.0 für das Lernen der neuen deutschen Rechtschreibung aus dem Buch „,Die neue Rechtschreibung *kurz und einfach” von Christian Stang mit der Richey und Klein Theorie. Diese Theorie besteht aus drei Phasen sind (1) die Planung, (2) die Herstellung des Produkts und (3) Die Bewertung und Verbesserung.

2. Das Ergebnis der Erstellung der Lernmedienmit der Hilfe von Macromedia Authorware 7.0 für das Lernen der neuen deutschen Rechtschreibung aus dem Buch ,Die neue Rechtschreibung *kurz und einfach” von Christian Stang sind:

a. Das Thema „Die neue Rechtschreibung kurz und einfach”, was die 6 Themen umfasst: 1. Die Laut-Buchstaben-Zuordung, 2. Die Fremdwortschreibung, 3. 
Die Worttrennung am Zeilenende, 4. Die Getrennt- und Zusammenschreibung, 5. Die Schreibung mit Bindestrich und 6. Die Groß- und Kleinschreibung.

b. Die Erstellung dieser Lermedien kann für das Lernen der neuen deutschen Rechtschreibung verwendet werden.

c. Diese Untersuchung hat einige Bilder und Texte.

d. Die Lernmedienmit Macromedia Authorware 7.0 Software wurden von einem Prüfer korrigiert. Nach der Validierung wird das Lernmedium mit der Hilfe Macromedia Authorware 7.0 Software verbessert. Das bedeutet, dass die Materialien der Lernmedien schon korrigiert wurde.

\section{LITERATURVERZEICHNIS}

Arsyad, Azhar. 2013. Media Pembelajaran. Jakarta: Raja Grafindo Pustaka.

Daryanto. 2010. Media Pembelajaran. Bandung: Satu Nusa.

Dittmann, Jürgen. 2008. Taschen Guide - die neue Rechtschreibung. München: Haufe Verlag.

Holst, Jan Henrik. 2005. Einheitlichkeit der Rechtschreibung als notwendiges Ziel. http://www.janhenrikholst.de/indey3.htm, gelesen am 27. Februar 2017 um 11.00 .

Lima Sekawan, Adie. 2007. EYD Plus. Jakarta: Limas.

mobil.derstandard.at/2978298/Ab-1-August-neue-Rechtschreibung-in-Deutschland--2008-in-Oesterreich, gelesen am 27. Februar 2017 um 10.00.

Schroeder, Frank. 2017. Die neue deutsche Rechtschreibung. https://www.betriebsausgabe.de/magazin/die-neue-deutsche-rechtschreibung4000/,gelesen am 28. Februar 2017, um 08.00.

Setyono,Budidkk.2008.ComputerAssistedInstructionMediaPembelajaranBerbasis MacromediaAuthorware6.0. Yogyakarta: Penerbit Ardana Media.

Stang, Christian. 2014. Die neue Rechtschreibung *kurz und einfach. Köln: Anaconda Verlag GmbH. 
Sugiyono. 2016. Metode Penelitian \& Pengembangan Research and Development. Bandung: Alfabeta.

Superdaf. 2012. Die 10 wichtigsten Regeln zur jetzt gültigen neuen Rechtschreibung.https://superdaf.wordpress.com/2012/07/13/die-10wichtigstenregeln-zur-jetzt-gultigen-neuen-rechtschreibung/, gelesen am 28. Februar 2017, um 09.00

TimWahanaKomputer.2004.MembuatAplikasiTutorialInteraktifDenganMacromediaA uthorware.Jakarta: Salemba Infotek.

Fitri Lasmaria Br Sihite is an alumni of German language study program of Unimed, Medan. 
Studia: Journal des Deutschsprogramms p-ISSN 2301-6108 e-ISSN 2654-9573

https://jurnal.unimed.ac.id/2012/index.php/studia/index 
Studia: Journal des Deutschsprogramms p-ISSN 2301-6108 e-ISSN 2654-9573

https://jurnal.unimed.ac.id/2012/index.php/studia/index 